\title{
Medical thoracoscopy in intensive care unit
}

\author{
Sanket Thakore ${ }^{1}$, Abdul Hamid Alraiyes ${ }^{2}$, Fayez Kheir ${ }^{3}$ \\ ${ }^{1}$ Division of Pulmonary Critical Care and Sleep Medicine, Yale School of Medicine, New Haven, CT, USA; ${ }^{2}$ Department of Pulmonary, \\ Interventional Pulmonology, Rosalind Franklin University of Medicine and Science, North Chicago, IL, USA; ${ }^{3}$ Division of Pulmonary Diseases, \\ Critical Care and Environmental Medicine, Tulane University Health Sciences Center, New Orleans, LA, USA \\ Contributions: (I) Conception and design: None; (II) Administrative support: None; (III) Provision of study materials or patients: None; (IV) \\ Collection and assembly of data: None; (V) Data analysis and interpretation: None; (VI) Manuscript writing: All authors; (VII) Final approval of \\ manuscript: All authors. \\ Correspondence to: Fayez Kheir, MD, MSc. Associate Professor of Medicine, Director of Interventional Pulmonary, Division of Pulmonary Diseases, \\ Critical Care and Environmental Medicine, Tulane University Health Sciences Center, New Orleans, LA, USA. Email: fkheir@tulane.edu.
}

\begin{abstract}
During the last decade, there has been a tremendous effort towards making procedures less invasive, which could reduce complications, decrease hospital stay and minimize overall health care cost. Medical thoracoscopy (MT) or pleuroscopy is a minimally invasive procedure commonly performed by interventional pulmonologist in United States. It has a favorable safety profile allowing access to the pleural cavity with a thoracoscope via a small chest wall incision to perform diagnostic or therapeutic interventions under direct visualization. MT allows the physician to perform pleural biopsy with high accuracy, drain loculated pleural effusion, guide chest tube placement and perform pleurodesis. As compared to videoassisted thoracoscopic surgery (VATS), MT is less invasive, does not require single lung ventilation, has a comparable diagnostic yield, and better tolerated in high-risk patients. MT can also be performed at bedside in critically ill patients. Although MT is generally safe, a multi-disciplinary discussion between the interventional pulmonologist, intensive care team, anesthesiologist and thoracic team is necessary to ensure best clinical practice as well as minimize complications for such high-risk patients. The purpose of this article is to review technique, diagnostic and therapeutic indications, as well as contraindications of performing bedside MT in intensive care unit. It aims to review both advantages and limitations of performing MT in intensive care unit.
\end{abstract}

Keywords: Medical thoracoscopy (MT); intensive care unit (ICU)

Submitted Jan 18, 2020. Accepted for publication May 04, 2020.

doi: 10.21037/jtd-2019-ipicu-02

View this article at: http://dx.doi.org/10.21037/jtd-2019-ipicu-02

\section{Introduction}

Medical thoracoscopy (MT) is a minimally invasive procedure, usually performed under local anesthesia or moderate sedation, where a thoracoscope is introduced into pleural cavity via a small chest wall incision allowing diagnostic and therapeutic intervention under direct visualization.

With recent growth in the field of interventional pulmonology and development of flexible-rigid thoracoscope, MT has become one of the common procedures performed by interventional pulmonologist (1-3). Nearly all MT are performed as an elective procedure either inpatient or outpatient setting. Depending on local resources, it can be performed in bronchoscopy suite or operating room. However, in certain scenarios, MT may need to be performed in intensive care unit (ICU).

The solitary idea behind MT is to introduce a camera via chest wall into the pleural cavity to create a platform that allows performing different interventions. There are two major types of MT: rigid and flexible-rigid (Figure 1).

Rigid thoracoscopy has been the traditional way of performing MT. It utilizes a rigid optic telescope along with rigid suction, and biopsy forceps. The first commercially 
A

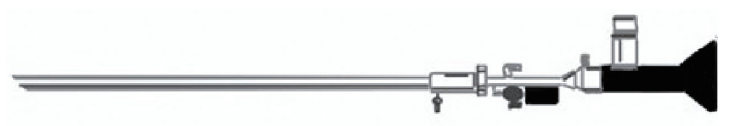

B

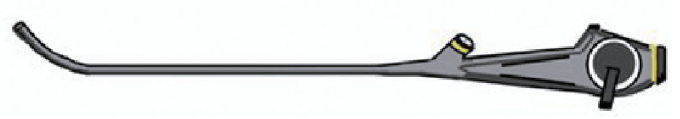

Figure 1 Available medical thoracoscopies. (A) Rigid thoracoscope, (B) flex-rigid.

available flexible-rigid thoracoscope was developed in the late 1990s as an alternative to the traditional rigid scope, offering better maneuverability and improved visualization (4).

A commonly used flexible-rigid thoracoscope by pulmonologists in United States is LTF-160 (Olympus, USA). It has an outer diameter of $7 \mathrm{~mm}$ with $2.8 \mathrm{~mm}$ working channel, total length of $27 \mathrm{~cm}(22 \mathrm{~cm}$ rigid shaft with $5 \mathrm{~cm}$ flexible tip), as well as angulation range of $160^{\circ}$ upwards to $130^{\circ}$ downwards.

While studies have demonstrated similar diagnostic yield and safety profile between rigid and flexible-rigid thoracoscopy $(5,6)$, there are proposed advantages of the latter. The handle of flexible-rigid thoracoscopy ergonomically fits into hand in a similar way to bronchoscope, which provides sense of familiarity to pulmonologist. Operator can pass all the familiar diagnostic and therapeutic tools commonly used in bronchoscopy, such as biopsy forceps, cryoprobe, argon plasma coagulation probe into the $2.8 \mathrm{~mm}$ working channel of flexible-rigid thoracoscope. In addition, flexible-rigid thoracoscope flexibility allows operator to maneuver around the non-deflated part of lung or dense adhesions, with ability to retroflex allowing to take biopsy near the insertion site of the trocar. In addition, the thoracoscope is compatible with the same Olympus tower for light and power source commonly used for bronchoscopy. However, rigid thoracoscope has the advantage of taking a larger biopsy specimen, albeit similar diagnostic yield, more efficient in breaking down dense adhesions as well as higher visual analog scale for ease of taking biopsies for the operator as compared to flexiblerigid thoracoscope (7).

Indications for MT include: parietal pleural biopsies for exudative pleural effusion of unclear etiology and/or pleural nodule/mass, chemical and mechanical pleurodesis under direct visualization, and mechanical adhesiolysis in complicated parapneumonic effusion $(1,8,9)$. Routinely in United States, MT are performed as an elective procedure either in bronchoscopy suite or operation room depending on the hospital policy. There is an increasing evidence in the medical literature that MT can safely and effectively performed under local anesthesia with none to minimal sedation (10-14).

The presence of pleural effusion in critically ill patients is common $(7.7 \%)$ and is associated with increased mortality, ICU and hospital stay, as well as need and duration of mechanical ventilation, regardless of disease severity in a large cohorts of ICU database (15). Recently, MT has been utilized in management for critically ill patients in the ICU with undiagnosed exudative pleural effusion (11).

Indications for performing MT at bedside in ICU are similar to indications for MT in general as described above. There are two main criteria that must be met before considering MT in ICU:

(I) Patient is critically ill and cannot undergo general anesthesia and surgery, and/or is unstable to be transported safely to operating room or endoscopy suite;

(II) MT would change management and outcome for the patient.

Most common reasons why we have performed MT in ICU are improving drainage of complicated parapneumonic effusion, pleural biopsies in pleural effusion of unclear etiology and persistent air leak. Relative contraindications are severe hypoxemia, stage 3 empyema, uncorrectable coagulopathy, history of ipsilateral pleurodesis, and extensive involvement of contralateral lung.

\section{Technique of bedside medical thoracoscopy in intensive care unit}

The technique of MT has been previously extensively described by our team (1). Below, is a summary along with few points while performing such procedure in the ICU:

\section{Equipments}

(I) Ultrasound with phased array transducer and sterile sheath;

(II) Sterile drape and surgical attire;

(III) $1 \%$ lidocaine;

(IV) Alcohol-based skin-sterilizing solution;

(V) Chest tube insertion kit (e.g., PleuraGuide) and 


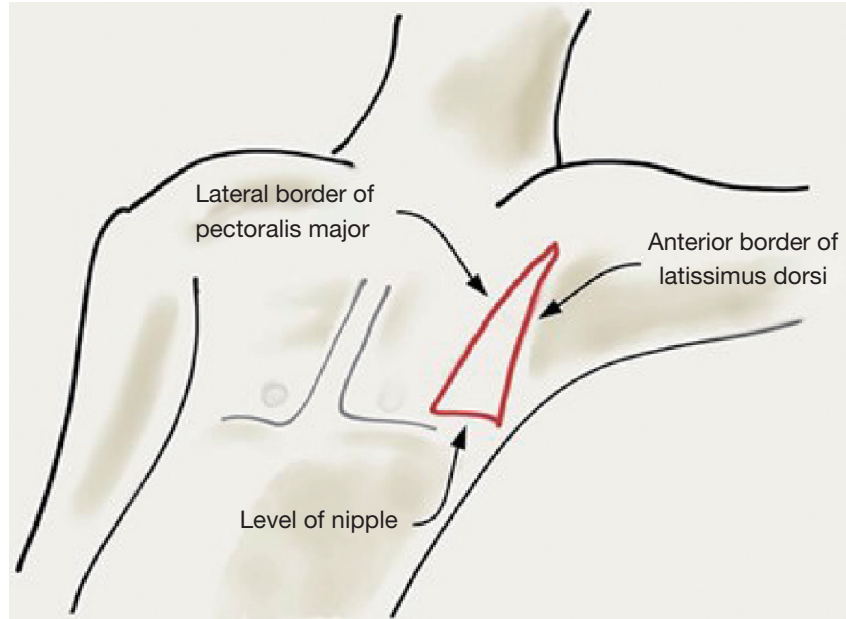

Figure 2 Triangle of safety superior to horizontal line at the nipple level, anterior to anterior border of Latissimus dorsi and posterior to lateral border of Pectoralis major.

(VI) Trocar (e.g., Olympus Flexible Trocar 8 mm MAJ1058, Covidien Mini Step Auto Suture Dilator and Cannula, 7-8 mm),

(VII) Flex-rigid thoracoscope (e.g., Pleuravideoscope LTF-160, Olympus);

(VIII) Monitor and processing tower.

\section{Patient position, monitoring and personnel}

Patient positioning for bedside MT in ICU can be challenging, but manageable with adequate personnel and equipment. One need to be mindful of all the lines, tubes, masks, and should consult with patient's nurse and attending physician for possible challenges. We usually perform MT while patient is on lateral decubitus position. It is difficult to perform MT in supine position, as maneuvering thoracoscope would be difficult and limited for operator. We tend to access the chest wall cavity guided by ultrasound image minimizing turning critically ill patients as much as possible. The patient should be placed closer to the edge of the ICU bed for operator's comfort, as ICU bed are much wider compared to operating room bed. The patient's vital signs should be continuously monitored on standard ICU monitors. We recommend at least two additional trained health care personnel to assist, patient monitoring and medication administration. We use cefazolin $1 \mathrm{~g}$ IV 30 to 60 minutes before MT for surgical infection prophylaxis, unless patients are already on antibiotics.

\section{Procedure}

(I) Ensure adequate positioning and monitoring of the patient as described above.

(II) Ensure adequate respiratory support as per patient's requirement from supplemental $\mathrm{O}_{2}$ to mechanical ventilator support.

(III) Site of entry into pleural cavity,

i. Identify largest fluid pocket based on thoracic ultrasound or;

ii. Triangle of safety (Figure 2): superior to horizontal line at the nipple level, anterior to anterior border of Latissimus dorsi and posterior to lateral border of Pectoralis major.

(IV) Operative team members clean their hands using standard surgical scrub technique and put on sterile gown and gloves.

(V) Large area of skin around the site of entry is prepared with chlorhexidine gluconate applicator. A sterile drape should be placed over the patient leaving small area around the entry site exposed.

(VI) Prepare procedural tray with all the necessary instruments as described above in sterile manner.

(VII) Inject $1 \%$ lidocaine at the entry site in adequate amount to anesthetize the skin, parietal pleural. In addition, lidocaine should be injected at the inferior rim of the upper and the superior rim of the lower rib to anesthetize the intercostal nerves as well as the periosteum of the ribs. Total dose of lidocaine should not exceed $3 / \mathrm{mg} / \mathrm{kg}$ body weight to avoid systemic toxicity (16).

(VIII) Approximately $1 \mathrm{~cm}$ horizontal incision through the skin and the subcutaneous tissue should be made by scalpel on the entry site in the middle of the intercostal space. The incision should be large enough got the trocar to fit in.

(IX) Blunt dissection utilizing blunt dissecting forceps, like curved Kelly hemostatic clamp, should be performed through the chest wall into the pleural cavity. Penetration though the parietal pleura into pleural cavity should occur over the superior rim of the lower rib.

(X) Blunt tip trocar of size 7 to $8 \mathrm{~mm}$ should be inserted into pleural cavity through the incision utilizing corkscrew method.

(XI) Remove obturator and insert flex-rigid thoracoscope into pleural cavity to perform intended diagnostic or therapeutic procedure. 
(XII) Administer midazolam or fentanyl intravenously in small doses as needed during the procedure.

(XIII) After the intended procedure is over, remove flexrigid thoracoscope and the trocar, and insert chest tube into the pleural cavity. Chest tube should be connected to chest drainage system with continuous negative suction to drain air and/or remaining pleural effusion.

(XIV) Chest tube size should be determined based on size of the trocar used. For 7 to $8 \mathrm{~mm}$ trocar, we routinely use 20-24 F chest tube.

\section{Complications of medical thoracoscopy in ICU}

MT with local anesthesia in combination with intravenous sedative medications is a relatively safe procedure. British thoracic society reported mortality of $0.34 \%$ based on combined data from 47 studies looking at 4,736 cases (10). They reported no mortality from MT. Cumulative rate of major complications (empyema, hemorrhage, port site tumor growth, bronchopleural fistula, postoperative pneumothorax or air leak and pneumonia) and minor complications (subcutaneous emphysema, minor hemorrhage, operative skin site infection, hypotension during procedure, raised temperature, atrial fibrillation) were reported to be $1.8 \%$ and $7.3 \%$ respectively. Complications related to bedside MT are very similar (10).

A recently published case series of 25 patients of bedside medical thoracoscopy in intensive care unit reported no major complications i.e., bleeding, or procedure related death. Most common minor complication reported was wound pain (24\%), followed by subcutaneous emphysema $(12 \%)$, transient hypotension (4\%), and incision site infection (4\%). No MT needed to be prematurely abandoned due to patient's instability (17).

An important limitation of performing bedside MT in ICU is performing procedure with staff who is not familiar with the routine. However, this can be easily overcome by team hurdle before the procedure and explain everyone in the team their role and expectations.

\section{Clinical cases for MT performed at ICU bedside}

We will present below few examples of cases of MT that are performed at the bedside in the ICU. However, it is important to recognize that such cases should be only performed after a multi-disciplinary discussion among different teams, adequate local expertise with MT and high level of coordination as well as comfort level among team members performing such procedures at the bedside.

\section{Complicated parapneumonic effusion}

A 38-years-old man who was critically ill man due to severe pleural infection and acute respiratory failure was transferred to our center for further management. $\mathrm{He}$ had multiple medical comorbidities including extreme obesity (BMI of 62), OSA, HTN and diabetes. Thoracic ultrasound showed multiloculated pleural effusion. He was on mechanical ventilation requiring low dose vasopressor. A large bore chest tube was inserted without adequate fluid drainage. Following multi-disciplinary discussion with thoracic surgery, intensive care and interventional pulmonary, he was deemed high risk for surgery by thoracic surgeons as well as unfit to move from ICU bedside due to his critical illness and extreme obesity. Furthermore, a discussion was made whether to proceed with intrapleural fibrinolytic therapy versus MT. Given the fact that he was extremely obese as well as the local expertise of our team with MT, a multi-disciplinary decision was made to perform a bedside thoracoscopy with the technique described above. Flexible-rigid thoracoscope was introduced into pleural cavity under sterile precautions without trocar, as trocar was not long enough due to his body habitus, through the same incision of his large bore chest tube after removal of the tube. First pleural fluid was suctioned. Then, with the help of pulmonary forceps, extensive mechanical adhesiolysis was performed followed by thorough irrigation of pleural cavity with sterile saline. Afterwards, a metallic tunneler was used to tunnel the fenestrated end of the indwelling pleural catheter (IPC) in inferomedial direction from first incision as per standard practice. Next, pulmonary forceps were passed through the working channel of the flexiblerigid thoracoscope and the fenestrated end of IPC was held with the pulmonary forceps. The thoracoscope was inserted, together with the IPC running outside parallel to the thoracoscope, into pleural cavity, and was advanced posteroinferiorly. Then, a sterilized pediatric bronchoscope was introduced through a $24 \mathrm{~F}$ chest tube and both were inserted together into the pleural cavity. With video assistance, chest tube was advanced posteroinferiorly, and bronchoscope was removed. This was followed by intrapleural fibrinolytic therapy for a total of 6 doses, and daily sterile water irrigation until $24 \mathrm{Fr}$ chest tube was removed on post procedure day 7 . He clinically improved and was transferred to long-term acute care facility 

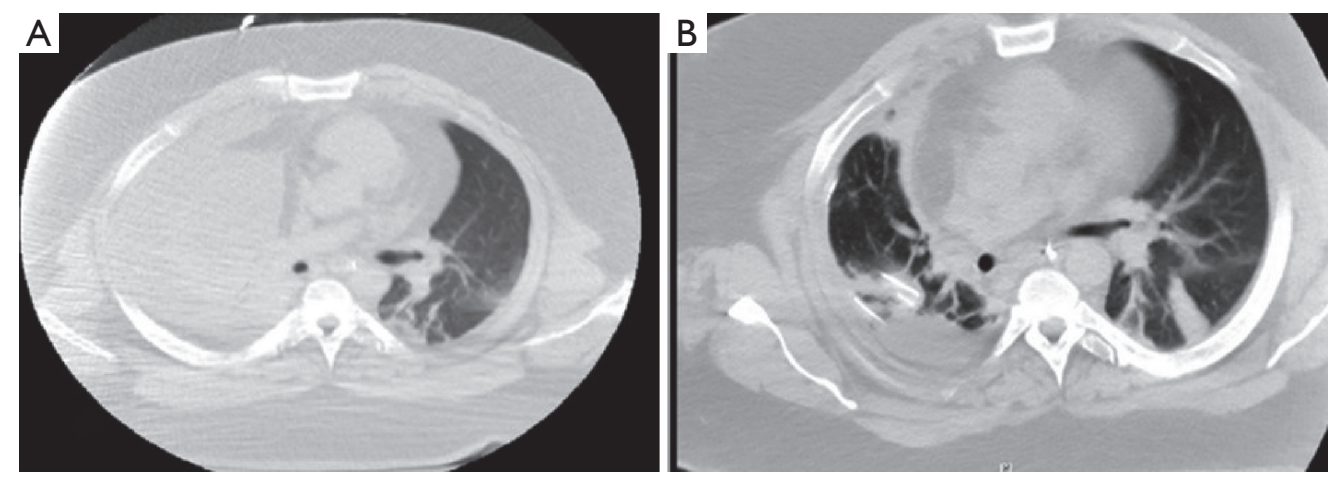

Figure 3 Computed tomography of the chest demonstrating significant improvement of the right pleural effusion (A) before medical thoracoscopy, (B) 1 week after medical thoracoscopy.
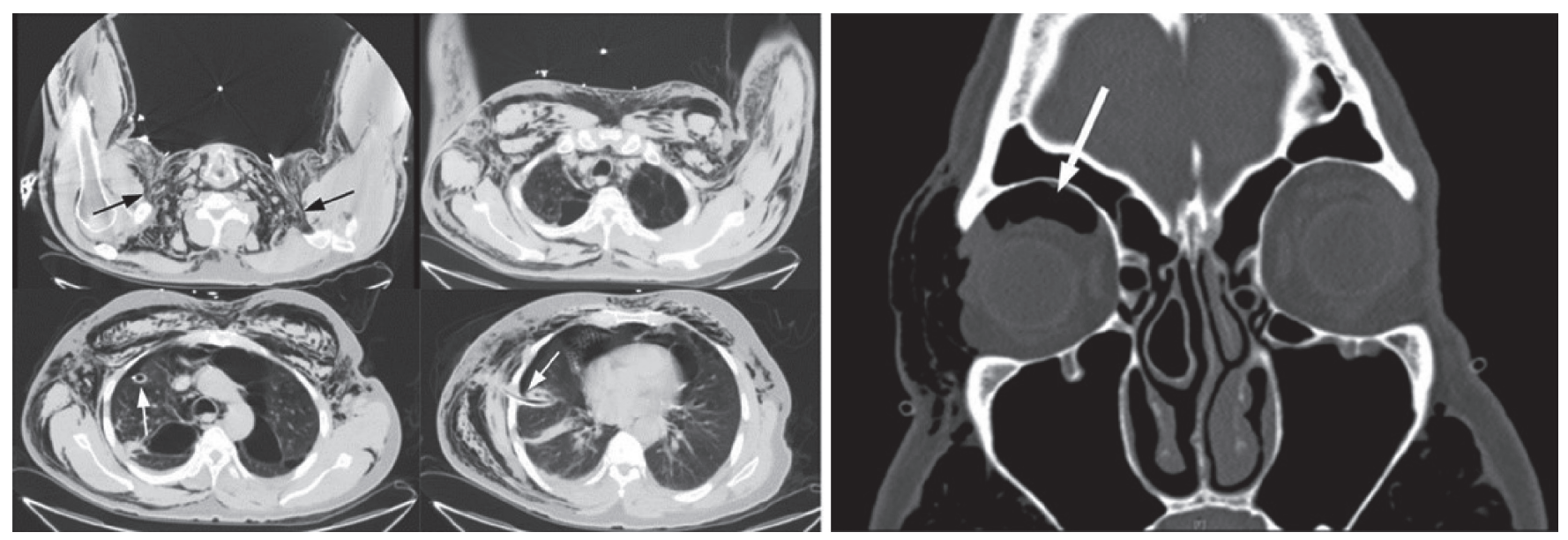

Figure 4 Computed tomography of the chest showing a severe subcutaneous emphysema (Black arrows) despite placement of chest tube (white arrows); head and neck computed tomography demonstrating subcutaneous orbital emphysema (arrow).

afterwards. His CT chest showed significant improvement of the right lower lobe consolidation and right pleural effusion (Figure 3). His IPC was removed 3 weeks later.

\section{Persistent air leak}

A 68-year-old man with history of severe emphysema/ COPD who was admitted to the ICU with secondary spontaneous pneumothorax along with severe subcutaneous emphysema, persistent air leak despite two chest tubes on continuous suction and increased right intraocular pressure due to subcutaneous orbital emphysema (Figure 4). Patient was on high flow nasal cannula. Due to his multiple comorbidities, a multi-disciplinary discussion among different specialties (interventional pulmonary, thoracic surgery, anesthesia and intensive care) was done and decision was made to proceed with a therapeutic procedure at the bedside in the ICU. This case represented a real challenge as he had severe spontaneous pneumothorax associated with increased intraocular pressure. Furthermore, secondary spontaneous pneumothorax represents a challenge, as there is high risk of recurrence if a bronchoscopic approach to stop or minimize air leak was not complemented by a thoracoscopic approach to prevent recurrence or identify air leak site. Given such unique challenge, we decided to proceed with both approaches consecutively if the air leak did not cease almost completely with initial bronchoscopic approach. He underwent a bronchoscopy in the ICU and a custom-made silicone plug along with fibrin glue was inserted into the target airway segment (Figure 5) with $<50 \%$ decrease in air leak. This was followed subsequently by MT with chemical pleurodesis, fibrin glue and visually 

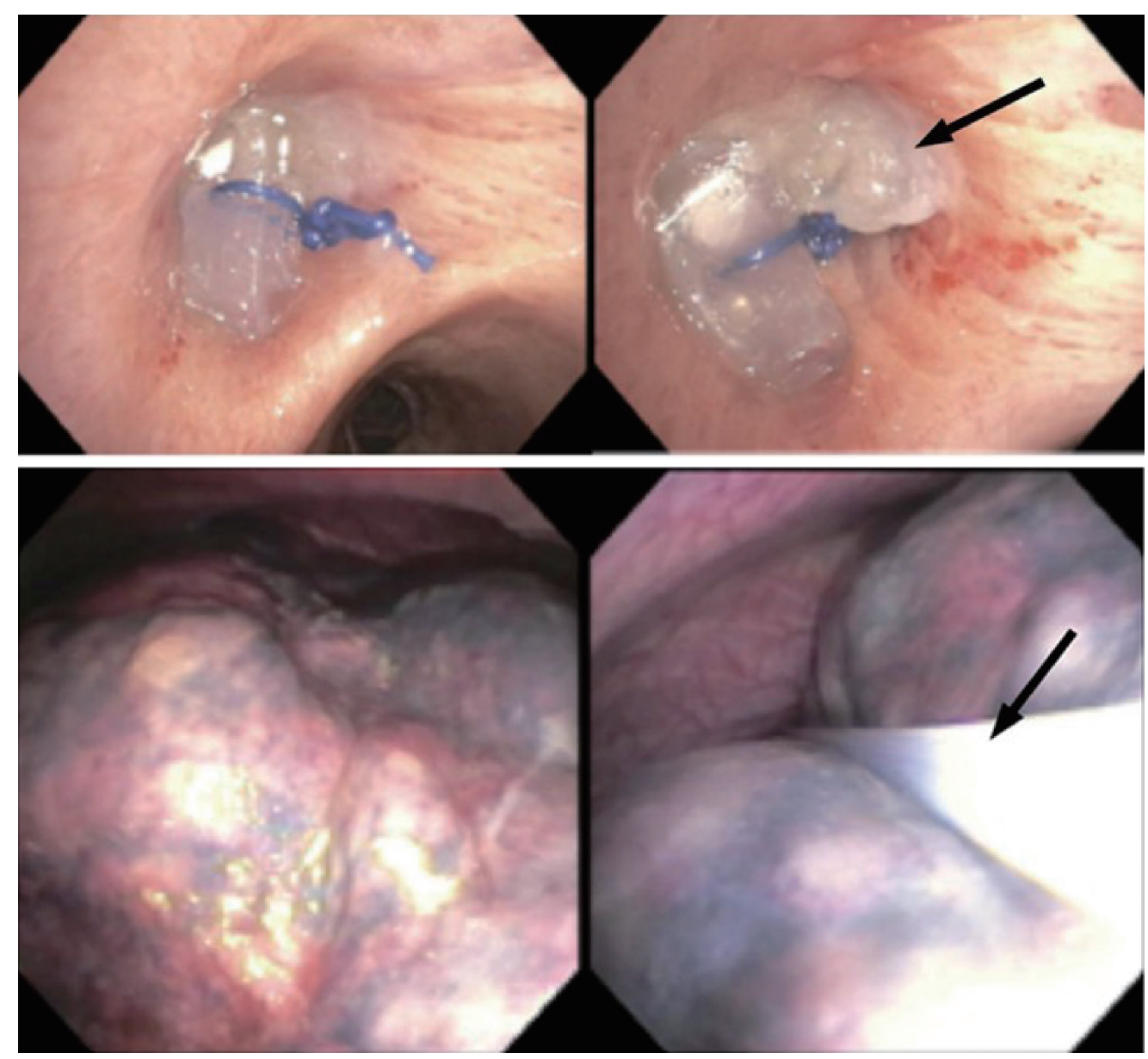

Figure 5 Bronchoscopic view of a custom-made silicone plug along with fibrin glue (arrow) deployed in the targeted airway; medical thoracoscope view allowing chemical pleurodesis, fibrin glue insertion and visually directed chest tube placement (arrow).

directed chest tube placement (Figure 5). Patient had immediate tremendous decrease in air leak followed by chest tube removal 3 days later (Figure 6). Four weeks later, he underwent an outpatient bronchoscopy for silicone plug removal (Figure 6).

\section{Exudative pleural effusion of unknown etiology}

A 78-year-old man had history of severe heart failure and right pleural effusion which was exudative along with negative cytology twice on repeated thoracentesis. $\mathrm{He}$ was admitted to ICU for increased dyspnea and close monitoring. A MT was done at the ICU bedside with minimal sedation and local anesthesia. Upon inspection, a pleural mass was noted on the diaphragm (Figure 7). A biopsy was done that was consistent with lung adenocarcinoma. Patient had a trapped lung and IPC was placed following MT. Such quick bedside procedure allowed the ICU team to discuss prognosis as well as diagnosis for such patient with unexplained exudative pleural effusion.

This case illustrates the importance of MT in undiagnosed exudative pleural effusion. Although the etiology of almost $80 \%$ of pleural effusions can be detected through careful history, physical examination as well as diagnostic thoracentesis, the remainder remain undiagnosed (10).

The advantage of MT is the ability to provide direct visualization of the pleural space with targeted pleural biopsies leading to high diagnostic yield in unexplained exudative pleural effusion $(18,19)$.

\section{Approach to pleural infection}

Figure 8 illustrates an algorithm to approach patients with pleural infection. Patients with small anechoic pleural 

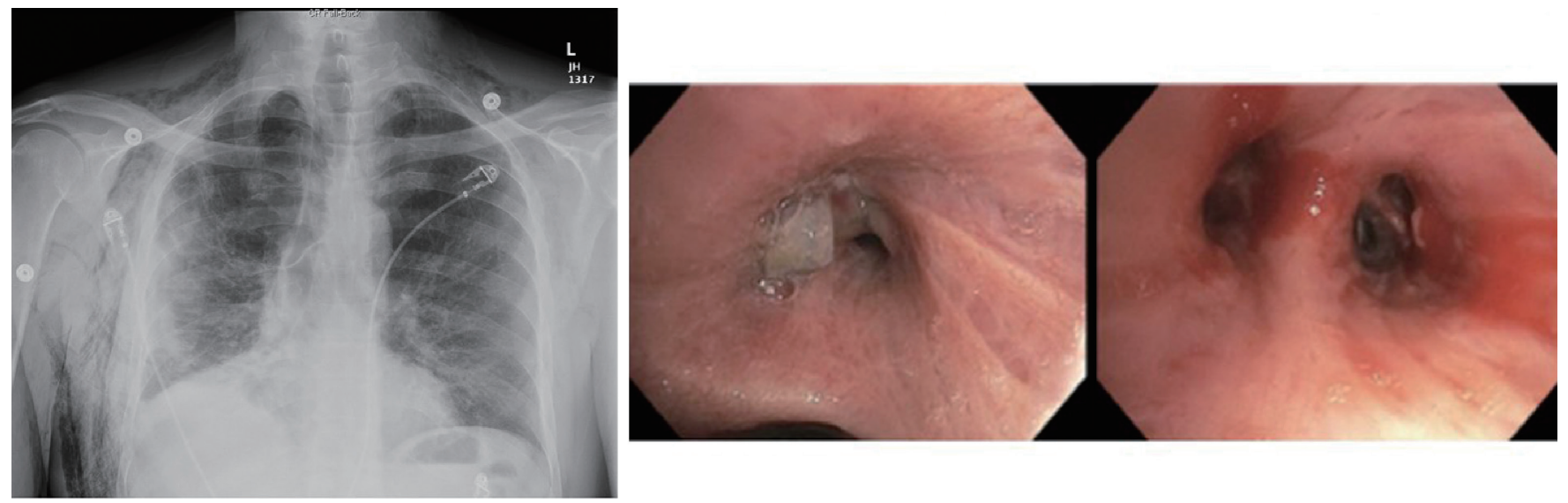

Figure 6 Chest radiography showing improvement of the air leak with subsequent removal of chest tubes; bronchoscopy view demonstrating a successful removal of the silicone plug few weeks later.
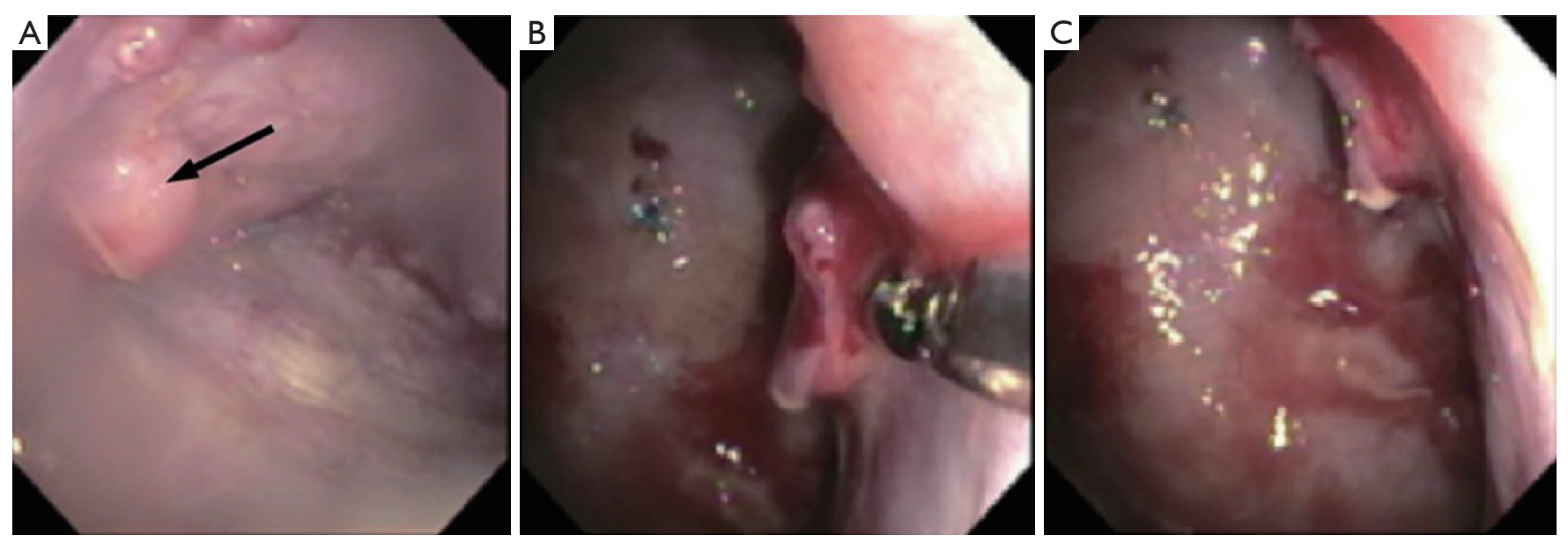

Figure 7 Medical thoracoscopy showing (A) a plural mass on the hemidiaphragm (arrow), (B,C) biopsy of the pleural mass.

effusion in setting of infection, usually resolves with medical therapy alone. If patient fails to respond to medical management, a chest tube insertion or serial thoracentesis would be needed. In patients with large anechoic pleural effusion or complex non-septated pleural effusion, either a thoracentesis or chest tube thoracostomy along with appropriate medical therapy should be performed, which ideally is decided after mutual discussion with patient. In patients with complex septated parapneumonic effusion, chest tube thoracostomy is recommended followed immediately by either fibrinolytic therapy or MT depending on patient comorbidities, local expertise and patient/ physician preference. If patient is critically ill and unstable for transfer to operating room or bronchoscopy suite, then bedside MT might be considered. If imaging shows trapped lung, echoic pleural effusion or stage 3 empyema, consider early thoracic surgery consultation following chest tube thoracostomy if patient is a candidate for surgery. If patient develops chronic pleural infection, then open thoracotomy is generally required (20). If not a surgical candidate, then non-surgical alternatives include tunneled pleural catheter (21), empyema tube (22), open pleural window, wound VAC or thoracoplasty (20).

When collecting pleural fluid, blood culture bottles for bedside inoculation of pleural fluid (23) or bedside ultrasound guided pleural biopsies (24) can be done to increase microbiological diagnostic yield. Pleural biopsies are performed with ultrasound assistance, using 18-gauge 


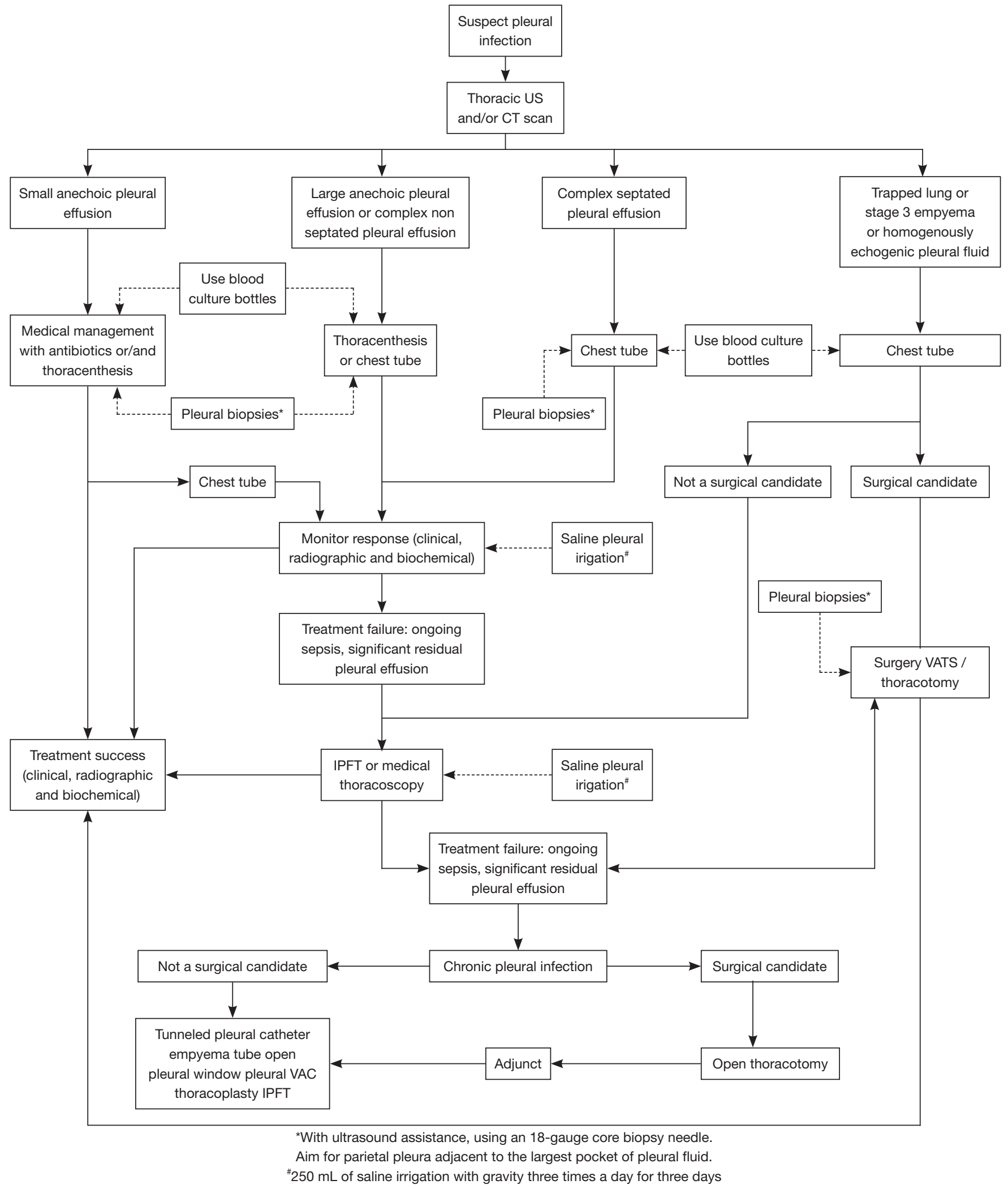

Figure 8 Approach to patients with pleural infection. US, ultrasound; IPFT, intrapleural fibrinolytic therapy; VATS, video-assisted thoracoscopic surgery; VAC, vacuum-assisted closure. 
core biopsy needle. We recommend aiming for parietal pleura adjacent to the largest pocket of pleural fluid. In addition, pleural irrigation with saline can be considered to improved pleural drainage (25). We recommend using study protocol, which is $250 \mathrm{~mL}$ of saline irrigation with gravity three times a day for three days (25).

\section{Conclusion}

In summary, MT can effectively be performed at bedside in selected patients in ICU by trained interventional pulmonologist. It can be a valuable if patients are at high risk for surgery and transportation. Flexible-rigid thoracoscope is preferable for performing bedside MT. The application of bedside MT in ICU includes pleural biopsy, adhesiolysis in pleural infection, and pleurodesis. Available evidence suggests that the safety profile of bedside MT in ICU is similar to MT performed in either operating room or bronchoscopy suite. More studies are needed to further define role and safety of bedside MT in ICU.

\section{Acknowledgments}

Funding: None.

\section{Footnote}

Provenance and Peer Review: This article was commissioned by the Guest Editors (Jonathan S. Kurman, Ashutosh Sachdeva and Rahul Nanchal) for the series "Interventional Pulmonology in the Intensive Care Unit Environment" published in Fournal of Thoracic Disease. The article has undergone external peer review.

Conflicts of Interest: All authors have completed the ICMJE uniform disclosure form (available at: http://dx.doi. org/10.21037/jtd-2019-ipicu-02). The series "Interventional Pulmonology in the Intensive Care Unit Environment" was commissioned by the editorial office without any funding or sponsorship. The authors have no other conflicts of interest to declare.

Ethical Statement: The authors are accountable for all aspects of the work in ensuring that questions related to the accuracy or integrity of any part of the work are appropriately investigated and resolved.

Open Access Statement: This is an Open Access article distributed in accordance with the Creative Commons Attribution-NonCommercial-NoDerivs 4.0 International License (CC BY-NC-ND 4.0), which permits the noncommercial replication and distribution of the article with the strict proviso that no changes or edits are made and the original work is properly cited (including links to both the formal publication through the relevant DOI and the license). See: https://creativecommons.org/licenses/by-nc-nd/4.0/.

\section{References}

1. Alraiyes A, Dhillon S, Harris K, et al. Medical Thoracoscopy: Technique and Application. Pleura 2016;3:2373997516632752.

2. Seijo LM, Sterman DH. Interventional pulmonology. N Engl J Med 2001;344:740-9.

3. Hooper CE, Lee YCG, Maskell NA. Setting up a specialist pleural disease service. Respirology 2010;15:1028-36.

4. Lee P, Colt HG. Rigid and semirigid pleuroscopy: the future is bright. Respirology 2005;10:418-25.

5. Agarwal R, Aggarwal AN, Gupta D. Diagnostic accuracy and safety of semirigid thoracoscopy in exudative pleural effusions: a meta-analysis. Chest 2013;144:1857-67.

6. Khan MA, Ambalavanan S, Thomson D, et al. A comparison of the diagnostic yield of rigid and semirigid thoracoscopes. J Bronchology Interv Pulmonol 2012;19:98-101.

7. Dhooria S, Singh N, Aggarwal AN, et al. A randomized trial comparing the diagnostic yield of rigid and semirigid thoracoscopy in undiagnosed pleural effusions. Respir Care 2014;59:756-64.

8. Murthy V, Bessich JL. Medical thoracoscopy and its evolving role in the diagnosis and treatment of pleural disease. J Thorac Dis 2017;9:S1011-21.

9. Hardavella G, Papakonstantinou NA, Karampinis I, et al. Hippocrates Quoted "If an Empyema Does Not Rupture, Death Will Occur": Is Medical Thoracoscopy Able to Make It Rupture Safely? J Bronchology Interv Pulmonol 2017;24:15-20.

10. Rahman NM, Ali NJ, Brown G, et al. British Thoracic Society Pleural Disease Guideline Group. Local anaesthetic thoracoscopy: British Thoracic Society Pleural Disease Guideline 2010. Thorax 2010;65 Suppl 2:ii54-60.

11. Ooi H. Bedside pleuroscopy in Taiwan: a great vision for critically-ill patients and intensivists. Biomedicine (Taipei) 2016;6:13.

12. Oldenburg FA Jr, Newhouse MT. Thoracoscopy. A safe, accurate diagnostic procedure using the rigid thoracoscope 
and local anesthesia. Chest 1979;75:45-50.

13. Rodriguez-Panadero F, Janssen JP, Astoul P.

Thoracoscopy: general overview and place in the diagnosis and management of pleural effusion. Eur Respir J 2006;28:409-22.

14. Loddenkemper R, Boutin C. Thoracoscopy: present diagnostic and therapeutic indications. Eur Respir J 1993;6:1544-55.

15. Bateman M, Alkhatib A, John T, et al. Pleural Effusion Outcomes in Intensive Care: Analysis of a Large Clinical Database. J Intensive Care Med 2020;35:48-54.

16. Laws D, Neville E, Duffy J. BTS guidelines for the insertion of a chest drain. Thorax 2003;58 Suppl 2:ii53-9.

17. Ooi H. Bedside pleuroscopy in the Intensive Care Unit. Ci Ji Yi Xue Za Zhi 2018;30:97-101.

18. Chen RL, Zhang YQ, Wang J, et al. Diagnostic value of medical thoracoscopy for undiagnosed pleural effusions. Exp Ther Med 2018;16:4590-4.

19. Hansen M, Faurschou P, Clementsen P. Medical thoracoscopy, results and complications in 146 patients: A retrospective study. Respir Med 1998;92:228-32.

20. Shen KR, Bribriesco A, Crabtree T, et al. The American

Cite this article as: Thakore S, Alraiyes AH, Kheir F. Medical thoracoscopy in intensive care unit. J Thorac Dis 2021;13(8):52325241. doi: 10.21037/jtd-2019-ipicu-02
Association for Thoracic Surgery consensus guidelines for the management of empyema. J Thorac Cardiovasc Surg 2017;153:e129-46.

21. Majid A, de Lima A, Parikh M, et al. Tunneled Pleural Catheters for Patients with Chronic Pleural Infection and Nonexpandable Lung. J Bronchology Interv Pulmonol 2019;26:132-6.

22. Biswas A, Jantz MA, Penley AM, et al. Management of chronic empyema with unexpandable lung in poor surgical risk patients using an empyema tube. Lung India 2016;33:267-71.

23. Menzies SM, Rahman NM, Wrightson JM, et al. Blood culture bottle culture of pleural fluid in pleural infection. Thorax 2011;66:658-62.

24. Psallidas I, Kanellakis NI, Bhatnagar R, et al. A Pilot Feasibility Study in Establishing the Role of UltrasoundGuided Pleural Biopsies in Pleural Infection (The AUDIO Study). Chest 2018;154:766-72.

25. Hooper CE, Edey AJ, Wallis A, et al. Pleural irrigation trial (PIT): a randomized controlled trial of pleural irrigation with normal saline versus standard care in patients with pleural infection. Eur Respir J 2015;46:456-63. 